\title{
CYBERSECURITY EDUCATION TO CREATE AWARENESS IN ARTIFICIAL INTELLIGENCE APPLICATIONS FOR DEVELOPERS AND END USERS
}

\author{
Maurice DAWSON \\ mdawson2@iit.edu
}

Annamaria SZAKONYI

annamaria.szakonyi@health.slu.edu

\author{
ILLINOIS INSTITUTE OF TECHNOLOGY, CHICAGO, UNITED STATES OF AMERICA \\ SAINT LOUIS UNIVERSITY, SAINT LOUIS, UNITED STATES OF AMERICA
}

\begin{abstract}
This new era brings new promises of technology that will bring economic and societal benefits. Artificial Intelligence is to be the disruptor for work and even military technological applications. However, developers and end-users will play keys roles in how this technology is developed and ultimately used. Among these two groups, there are cybersecurity concerns that need to be considered. In this paper, the researchers address the process of secure development and testing. Also, for the end-user appropriate methods, procedures, and recommendations are defined that can mitigate the overall use of this technology within an enterprise.
\end{abstract}

\section{KEYWORDS:}

Artificial intelligence, cybersecurity training, workforce development

\section{Introduction}

Artificial Intelligence (AI) has been a part of our everyday lives, yet often we ignore its prevalence in today's world. Some immediate AI associations may bring up images of robots and self-driving cars, but AI often presents itself in our lives in much simpler and more subtle ways. The spam filter in our email account, our online movie, and shopping product recommendations, the credit card theft alerts from our banking accounts, our online job recommendations, our ridesharing matches, and proposed routes, or even our web-based translation tools these are all based on artificial intelligence algorithms. They have played an essential role in the everyday things we do, and we take them for granted, without considering the potential security implications these technologies bring.

Traditional Software Development Life Cycle (SDLC) environments did not have cybersecurity embedded into the 
design lifecycle (Kumar \& Bhatia, 2014). Thus processes were developed and championed by organizations to integrate security into the lifecycle (Dawson, Burrell, Rahim \& Brewster, 2010). The shift in focus from systems to software has been rapidly occurring in the last decade, impacting developers. In the area of Artificial Intelligence (AI), this becomes vital as the system can be exploited and ultimately provide attackers unparalleled access to resources.

Researchers identify several AI failures, including imaging tagging for black people, inability to filter adult content, and issues associated with E-Assistant for ambulance assistance (Yampolskiy \& Spellchecker, 2016). A significant event was that the self-driving car ended in a driver killed while using autopilot while viewing a film (Levin \& Woolf, 2016). Previously researchers uncovered vulnerabilities in the software within the vehicles that did not have AI incorporated. So with the technology such as AI now embedded in some cars, the threat landscape has altered drastically, creating new opportunities for nefarious actors.

\section{Software testing}

Software testing is a misunderstood task that can make an application vulnerable and increase the threat landscape (Potter \& McGraw, 2004). As many of the system's vulnerabilities are found within the application layer, testing is critical to minimizing the overall system (Alhazmi, Malaiya \& Ray, 2007; Paul, 2015). Secure software testing is an essential task for developing an application that is not readily susceptible to attacks. In Figure no. 1, a process is displayed that enables developers to produce code that undergoes rigorous testing phases before deployment.

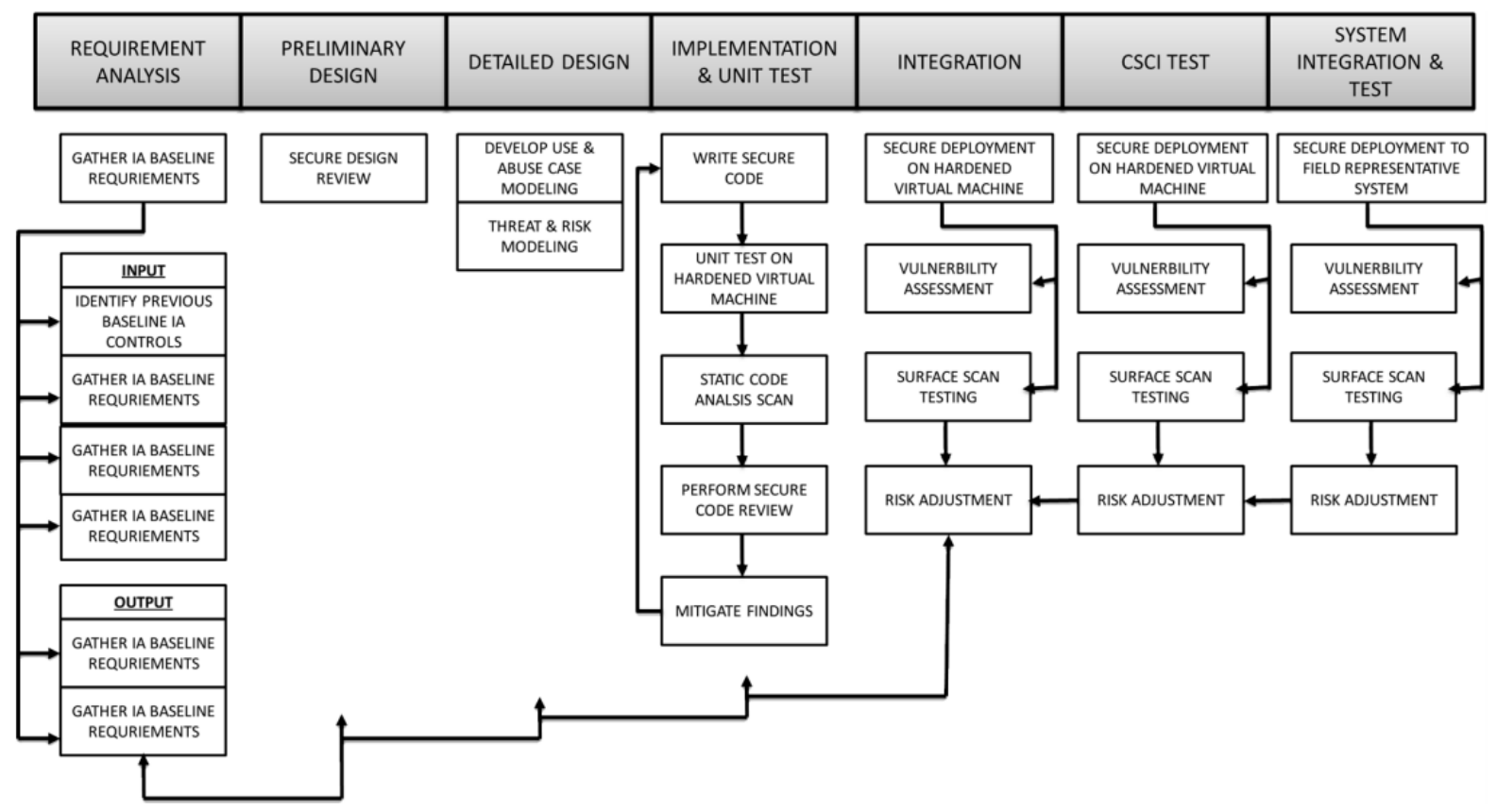

Figure no. 1: Secure Software Development Process

(Source: Authors)

In the requirement analysis phase, baseline requirements must be defined. All threshold requirements must be established that need to be met. This would also include how they are tested, such as inspection, observation, analysis, and an actual test. In the next phase, the application or system's preliminary design 
goes through a secure design review. This review goes through the requirements, expected system functionality. Once this phase is finished, the detailed design occurs where threat modeling, risk modeling, test cases, use, and abuse cases are developed. Only once this stage has met all criteria for moving forward, the implementation and unit test begins. Here secure code is written and unit testing on a hardened Virtual Machine (VM). The VM is hardened according to the environment the expected production unit will be in. For example, if it were the application needed to be placed into a federal system, then it would need to complete the National Institute of Standards and Technology (NIST) Special Publication (SP) 800-53 Security and Privacy Controls for Federal Information Systems and Organizations. This would mean that the VM has either low, medium, or highsecurity controls applied to the VM before the test begins. Once this occurs, static code analysis and code reviews start. Upon completion of these tasks, vulnerabilities are discovered and then mitigated down to an acceptable level. The integration phase would take the secure, hardened VM and perform a vulnerability assessment, surface scan, and risk adjustment. Afterward, there are two more testing phases: the Computer Software Configuration Item (CSCI) test and system integration and test. During these phases, the same subtasks occur as in integration.

\subsection{Sandbox environment}

Performing multiple tests in a sandbox environment allow time to discover any bug or vulnerability before a production release. This discovery process, through rigorous testing, minimizes the number of vulnerabilities that the end-user will have to inherit. Additionally, this allows the application to be isolated and in a safe environment, so if something does occur, it will not affect any other systems. Without knowing the application's full behavior in a production environment or one that is integrated with other methods, this sandbox would allow developers to add in those other systems while testing them incrementally slowly. If there is a failure, it is easy to identify the system environment or configuration in which the fault appears.

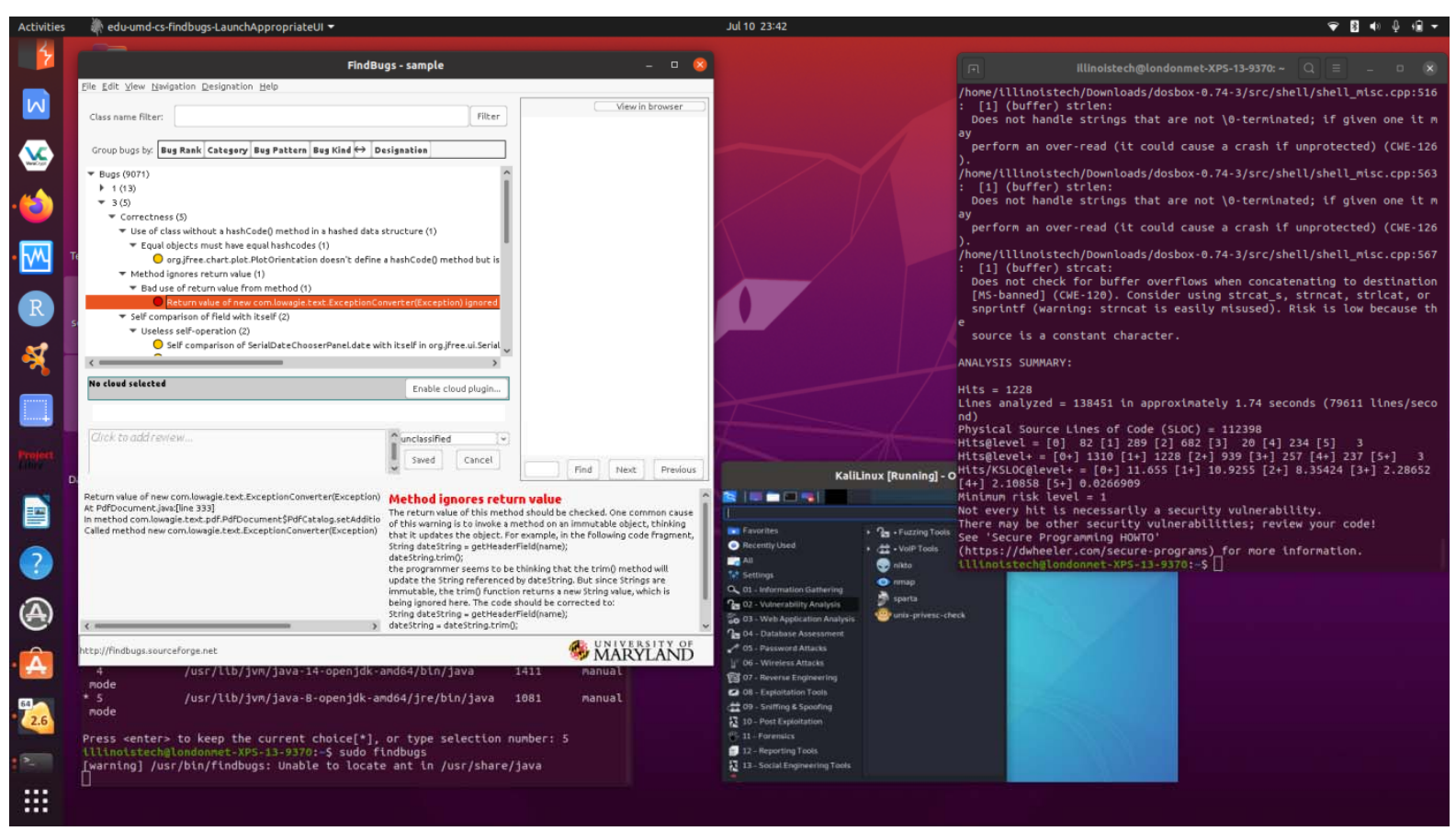

Figure no. 2: Linux Sandbox Environment 
The Secure Software Development Process is crucial for implementing secure and reliable products for end users. When this process is implemented properly, vulnerabilities arising from software security gaps can be mitigated, providing a high level of confidence for users. However, security does not stop where software development ends. Once users adopt the technology, there is a level of responsibility they must carry to protect their own devices and data.

\section{Security in the end user process}

The end-user must understand the relationship between cybersecurity and Artificial Intelligence. With the expansion of emerging technologies in our everyday lives, such as with the Internet of Things (IoT), our connected devices provide convenient ways to fulfill daily activities. With that convenience, however, there are many risks involved related to the security and privacy of data.

\subsection{Authentication and password management}

When AI is applied in the end-user experience, it generally serves the goals of customizations and personalization's for the end-user. For AI to work, therefore, accounts must be unique and personally identifiable to the specific user. Hence, authentication plays a vital role in the process. For online shopping or movie recommendations to properly match our needs, they must learn from our previous experiences with the system, and that data must be accessible for future use.

Password management is probably the most critical aspect of end-user security related to authentication of $\mathrm{AI}$ and Machine Learning (ML) systems. Developers and technologists can implement various controls and safeguards within their products, but today, "humans remain the weakest link in Internet security" (Tam, Glassman \& Vandenwauver, 2008). General best practices of passwords (long and complex, mixed case and symbols, regularly changed, etc.) are not always implemented even if well known. Users often use readily identifiable information, such as birth dates or nicknames, or write down essential passwords in an insecure location (Tam, Glassman \& Vandenwauver, 2008). Furthermore, the reuse of passwords is a common issue across various platforms (Jeslet, Sivaraman, Uma, Thangadurai \& Punithavalli, 2010). Researchers found that users "associate secure passwords with a loss of convenience (i.e., the security convenience tradeoff), and they do not want to give up convenience" (Tam, Glassman, \& Vandenwauver, 2008). This is an essential factor when considering efforts to increase awareness of secure password management benefits and the risks of breached accounts and information. Password managers are a possible way to balance that tradeoff towards both higher convenience and security for the user. Still, those also carry certain risks due to the developer efforts mentioned above. Multi-factor authentication can provide that much needed added protection as an additional layer of authentication mechanism, which users should implement associated with their sensitive accounts.

On the other hand, various AI techniques, such as deep learning, have been applied in cracking passwords, especially with password brute force attacks (Trieu, 2018). These tools make it extremely efficient for authentication mechanisms to be jeopardized, making it even more imperative for users to apply secure practices with their everyday accounts.

\subsection{Device hardening and settings}

The regular maintenance of personal devices is essential for a secure end-user experience. Device hardening is the process of eliminating vulnerabilities through security patches and disabling non-essential services and connectivities on independent systems. Devices without security patches installed can be vulnerable to outside 
attacks. This is especially important with everyday gadgets such as smartphones that are always connected to the Internet and transmit significant amounts of personal and sensitive data, such as banking application logins and personal email accounts. Therefore, it is imperative that users follow recommended patch schedules and immediately install device and application updates when made available.

Device settings can provide valuable protection from outside threats and the exploitation of user data. As an example, network and connectivity configurations that are always on (WiFi and Bluetooth) can massively increase the risks of attackers gaining access to a personal device, especially when other measures are not correctly in place for safeguards. Also, application-level permissions that are required at application installations are essential to consider. Research shows that only about $35 \%$ of users review these needed permissions, and about $11 \%$ of users never read these permissions (Alani, 2017; Breitinger, Tully-Doyle \& Hassenfeldt, 2020). When users overlook these permissions and install the apps for convenience, they share unnecessary access to their device features and data that is not justified for the specific application.

\subsection{Trust and privacy}

The final often overlooked aspect of end-user AI security is related to the actual data collected about the user. AI technologies require a level of trust by the consumer towards the technology provider, where the user voluntarily provides various types of data regarding their behaviors and technology use that can be translated to valuable insights to them. There is a silent contract between the user who provides their data in exchange for more customized, more convenient services and products. However, this brings the concern of the privacy-convenience tradeoff. Namely, the user's level of privacy is willing to give up in exchange for those services and insights. There are essential questions that must also be raised when considering these decisions. Are the customers genuinely aware of what is being collected from them? Our data indeed collected for the reasons, and only for those reasons, that the user consented to?

Users often consent to the "Terms and Conditions" page when signing up for new technology products or services, without truly considering what they agree to regard their data to be collected. Furthermore, organizations sometimes exploit users' unawareness, and partner with outside organizations or sell the collected data to marketers. Even if this may be beneficial to consumers for the higher customizability and personalization of the products and services they use, it raises valid questions on data ethics and privacy.

The ethical use of data has been a conversation in various policy level discussions across the globe. "Data collected today may (and most likely will) be used in unexpected ways tomorrow" (Steinebach \& Waidner, 2018). This is especially true for data residing on smart devices and wearables, such as smartphones and smartwatches, that are continuously connected for maximum efficiency. Other smart electronics and IoT devices, such as intelligent thermostats, collect valuable user data about everyday habits that can be used to benefit the user but can also be exploited if not configured correctly. Not to mention devices that transmit sensitive data, such as health behaviors or electronic health records, monitored by fitness trackers and medical devices, could result in adverse effects and even death if hijacked by attackers. Mining application and device usage patterns allow companies to understand people's interests and provide valuable insights into how they use their devices for potential personalizations and product improvements (Do \& Gatica-Perez, 2010). But sometimes, that personalization becomes too personal, and the consumer data is abused. 
There have been various examples of data misuse through AI methods. A case in the financial industry was CompuCredit \& Jefferson Capital, and its application of Machine Learning technologies to track their consumers' purchases on their credit cards. They then used this data to rate their customers' credit risks based on an undisclosed "behavior scoring model". They penalized consumers with reduced credit limits for using their cards for transactions such as bars, night clubs, marriage counseling, therapy, tire-repair services, pawnshops, or massage parlors (Federal Trade Commission v. CompuCredit Corporation and Jefferson Capital Systems, LLC, 2008).

Another all-too-common example is targeted advertising and social media. "Online Behavioral Advertising (OBA) refers to the practice of tracking users across websites to infer user interests and preferences" (Toubiana, Narayanan, Boneh, Nissenbaum \& Barocas, 2010). This type of advertising happens daily on our smartphones and applications installed on our smart devices. When data on consumer behavior merges with data in our social media accounts, marketers have easy access to pushing the content that may be interesting to us. Often, this is precisely what the consumer wants to see. But when a consumer's fitness activity is tracked and associated with their health information, insurance rates could rise when the activity levels are not as expected by the insurance provider. This raises the vital question of where the need for convenience stops and when data privacy is breached.

The privacy-convenience tradeoff could be displayed as a continuum that varies for each user. When the slider is moved more towards the convenience side of the continuum, the user must surrender some privacy expectations in exchange for a higher convenience level. When the slider crosses more towards the privacy side, the user sacrifices some of the technology's comforts for more data protection (see Figure no. 3). Some users want those data insights at a high level and don't mind allowing a broad view of their personal information. Others may prefer more privacy at the expense of less convenience. The important thing is that users must be aware of this tradeoff to make a conscious decision of where they prefer to be placed on the scale.

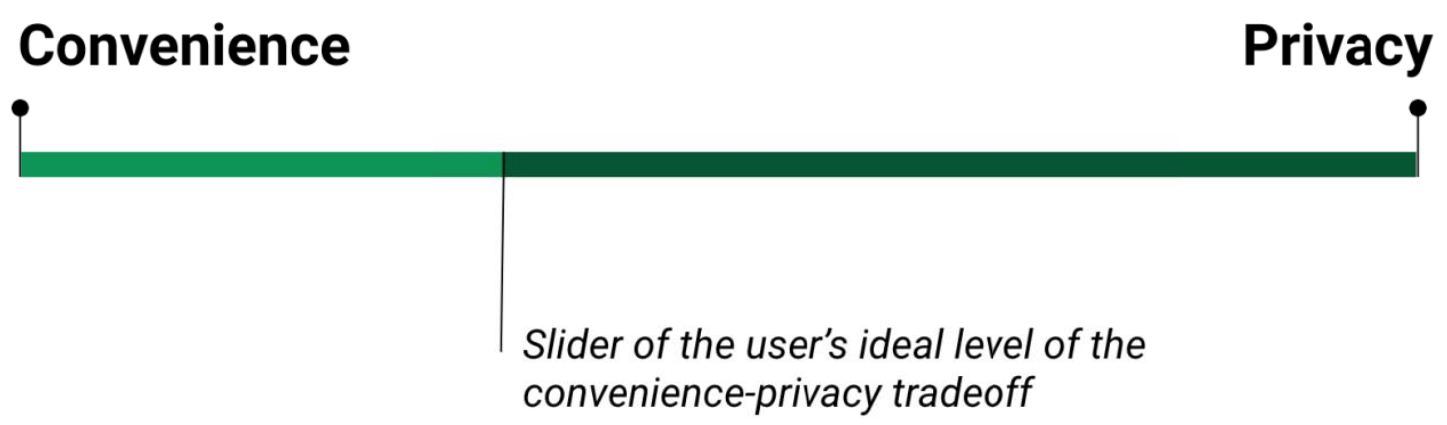

Figure no. 3: The Convenience-Privacy Continuum

(Source: Authors)

\section{Emerging computing environments}

To better deal with the emerging computing environments, frameworks need to be implemented that are suited to handle this change. Applying a holistic cybersecurity framework such as the Mission Framework would allow organizations seeking to establish environments that would enable them to be successful regardless of location while examining external and internal conditions (Dawson, 2018). This framework comprises three themes education, policy, and technology to implement cybersecurity within an organization (see Figure no. 4). 


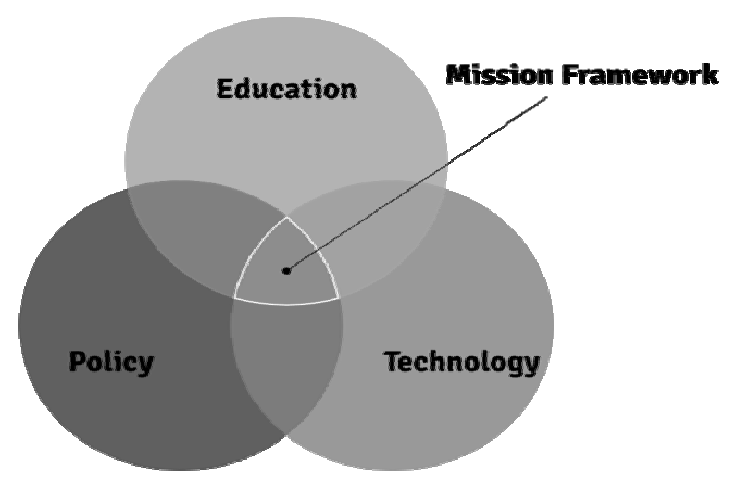

Figure no. 4: Mission Framework

(Source: Authors)

\section{Conclusions}

This paper explored areas of concern for developers and end-users to implement cybersecurity into AI effectively into organizations a few recommendations arise. Essentially, findings suggest that developers, having a secure development process is critical to ensure that vulnerabilities are mitigated that bad actors cannot take advantage of in the system. Second, proper testing ensures that the AI application works as intended and locates any problems before deployment. Rooting out integration issues is vital. These could be potential sources of new vulnerabilities as the threat landscape will change once the application becomes part of a more extensive system. Third, they need to be aware of what cybersecurity risks they may confront in their role as it relates to the system for the end-user. This may require customized training for specific functions that provide them insight into performing their job in the life cycle more securely. Future research will need to explore these cybersecurity awareness initiatives such as customized educational training programs sufficient for developers and end-users as organizations implement AI.

\section{REFERENCES}

Alani, M. M. (2017). Android users privacy awareness survey. International Journal of Interactive Mobile Technologies (IJIM), Vol. 11, Issue 3, 130-144.

Alhazmi, O. H., Malaiya, Y. K., \& Ray, I. (2007). Measuring, analyzing and predicting security vulnerabilities in software systems. Computers \& security, Vol. 26, Issue 3, 219-228.

Breitinger, F., Tully-Doyle, R., \& Hassenfeldt, C. (2020). A survey on smartphone user's security choices, awareness and education. Computers \& Security, Vol. 88.

Dawson, M. (2018). Applying a holistic cybersecurity framework for global IT organizations. Business Information Review, Vol. 35, Issue 2, 60-67.

Dawson, M., Burrell, D.N., Rahim, E., \& Brewster, S. (2010). Integrating software assurance into the software development life cycle (SDLC). Journal of Information Systems Technology and Planning, Vol. 3, Issue 6, 49-53.

Do, T. M. T., \& Gatica-Perez, D. (2010). By their apps you shall understand them: mining large-scale patterns of mobile phone usage. Proceedings of the $9^{\text {th }}$ International Conference on Mobile and Ubiquitous Multimedia, 1-10. 
Federal Trade Commission v. CompuCredit Corporation and Jefferson Capital Systems, LLC. (2008). Complaint for Permanent Injunction and Other Equitable Relief, available at: https://www.ftc.gov/sites/default/files/documents/cases/2008/06/080610compucreditcmplt.pdf, accessed on 20 June 2020.

Jeslet, D. S., Sivaraman, G., Uma, M., Thangadurai, K., \& Punithavalli, M. (2010). Survey on awareness and security issues in password management strategies. International Journal of Computer Science and Network Security, Vol. 10, Issue 4, 19-23.

Kumar, G., \& Bhatia, P. K. (2014). Comparative analysis of software engineering models from traditional to modern methodologies. $4^{\text {th }}$ International Conference on Advanced Computing \& Communication Technologies, 189-196.

Levin, S., \& Woolf, N. (2016). Tesla driver killed while using autopilot was watching Harry Potter, witness says, available at: https://www.theguardian.com/technology/2016/jul/ 01/tesla-driver-killed-autopilot-self-driving-car-harry-potter, accessed on 20 June 2020.

Paul, M. (2015). Official (ISC) ${ }^{2}$ Guide to the CSSLP. CRC Press.

Potter, B., \& McGraw, G. (2004). Software security testing. IEEE Security \& Privacy, Vol. 2, Issue 5, 81-85.

Steinebach, M., \& Waidner, M. (2018). Artificial Intelligence in IT Security. Whither Artificial Intelligence, 31-36.

Tam, L., Glassman, M., \& Vandenwauver, M. (2010). The psychology of password management: a tradeoff between security and convenience. Behaviour \& Information Technology, Vol. 29, Issue 3, 233-244.

Toubiana, V., Narayanan, A., Boneh, D., Nissenbaum, H., \& Barocas, S. (2010). Adnostic: Privacy preserving targeted advertising. Proceedings Network and Distributed System Symposium.

Trieu, K., \& Yang, Y. (2018). Artificial Intelligence-Based Password Brute Force Attacks. MWAIS Proceedings, Vol. 39.

Yampolskiy, R. V., \& Spellchecker, M. S. (2016). Artificial intelligence safety and cybersecurity: A timeline of AI failures, available at: https://arxiv.org/ftp/arxiv/ papers/1610/1610.07997.pdf, accessed on 20 June 2020 . 